\title{
Picard and Adomian methods for quadratic integral equation
}

\author{
EL-SAYED A.M.A. ${ }^{1}$, HASHEM H.H.G. ${ }^{2}$ and ZIADA E.A.A. ${ }^{2}$ \\ ${ }^{1}$ Faculty of Science, Alexandria University, Alexandria, Egypt \\ ${ }^{2}$ Faculty of Engineering, Mansoura University, Mansoura, Egypt \\ E-mails: amasayed@hotmail.com / hendhghashem@yahoo.com / \\ eng_emanziada@yahoo.com
}

\begin{abstract}
We are concerning with two analytical methods; the classical method of successive approximations (Picard method) [14] which consists the construction of a sequence of functions such that the limit of this sequence of functions in the sense of uniform convergence is the solution of a quadratic integral equation, and Adomian method which gives the solution as a series see ([1-6], [12] and [13]). The existence and uniqueness of the solution and the convergence will be discussed for each method.
\end{abstract}

Mathematical subject classification: Primary: 39B82; Secondary: 44B20, 46C05.

Key words: quadratic integral equation, Picard method, Adomian method, continuous unique solution, convergence analysis, error analysis.

\section{Introduction}

Quadratic integral equations (QIEs) are often applicable in the theory of radiative transfer, kinetic theory of gases, in the theory of neutron transport and in the traffic theory. The quadratic integral equations can be very often encountered in many applications.

The quadratic integral equations have been studied in several papers and monographs (see for examples [7-11] and [16-22]). 
The Picard-Lindelof theorem for the initial value problem

$$
x^{\prime}(t)=f(t, x(t)), \quad x(0)=x_{0}
$$

was proved in [14] and the solution can be shown as the limit of constructed sequence.

The existence of continuous solution of the nonlinear quadratic integral equation

$$
x(t)=a(t)+g(t, x(t)) \int_{0}^{t} f(s, x(s)) d s
$$

was proved in [18] by using Tychonov fixed point theorem where $f$ and $g$ satisfy Carathéodory condition.

In this work, we will prove the existence and the uniqueness of continuous solution for (1) by using the principle of contraction mapping. Also, we are concerning with the two methods; Picard method and Adomian method.

\section{Main Theorem}

Now, equation (1) will be investigated under the assumptions:

(i) $a: I \rightarrow R_{+}=[0,+\infty)$ is continuous on $I$ where $I=[0,1]$;

(ii) $f, g: I \times D \subset R_{+} \rightarrow R_{+}$are continuous and there exist positive constants $M_{1}$ and $M_{2}$ such that $|g(t, x)| \leq M_{1}$ and $|f(t, x)| \leq M_{2}$ on $D$;

(iii) $f$, $g$ satisfy Lipschitz condition with Lipschitz constants $L_{1}$ and $L_{2}$ such that,

$$
\begin{aligned}
|g(t, x)-g(t, y)| & \leq L_{1}|x-y|, \\
|f(t, x)-f(t, y)| & \leq L_{2}|x-y| .
\end{aligned}
$$

Let $C=C(I)$ be the space of all real valued functions which are continuous on $I$.

Define the operator $F$ as

$$
(F x)(t)=a(t)+g(t, x(t)) \int_{0}^{t} f(s, x(s)) d s, \quad \forall x \in C .
$$


Theorem 1. Let the assumptions (i)-(iii) be satisfied. If $h=\left(L_{1} M_{2}+\right.$ $\left.L_{2} M_{1}\right)<1$, then the nonlinear quadratic integral equation (1) has a unique positive solution $x \in C$.

Proof. It is clear that the operator $F$ maps $C$ into $C$.

Now define a subset $S$ of $C$ as

$$
S=\{x \in C:|x-a(t)| \leq k\}, \quad k=M_{1} M_{2} .
$$

Then the operator $F$ maps $S$ into $S$, since for $x \in S$

$$
|x(t)-a(t)| \leq M_{1} M_{2} \int_{0}^{t} d s=M_{1} M_{2} t=M_{1} M_{2} .
$$

Moreover it is easy to see that $S$ is a closed subset of $C$. In order to show that $F$ is a contraction we compute

$$
\begin{aligned}
(F x)(t)-(F y)(t)= & g(t, x(t)) \int_{0}^{t} f(s, x(s)) d s \\
& -g(t, y(t)) \int_{0}^{t} f(s, y(s)) d s+g(t, x(t)) \\
& \times \int_{0}^{t} f(s, y(s)) d s-g(t, x(t)) \int_{0}^{t} f(s, y(s)) d s \\
= & {[g(t, x(t))-g(t, y(t))] \int_{0}^{t} f(s, y(s)) d s } \\
& +g(t, x(t)) \int_{0}^{t}[f(s, x(s))-f(s, y(s))] d s \\
|(F x)(t)-(F y)(t)| \leq & |g(t, x(t))-g(t, y(t))| \int_{0}^{t}|f(s, y(s))| d s \\
& +|g(t, x(t))| \int_{0}^{t}|f(s, x(s))-f(s, y(s))| d s \\
\leq & L_{1} M_{2}|x-y|+L_{2} M_{1} \int_{0}^{t}|x(s)-y(s)| d s \\
||(F x)(t)-(F y)(t) \| \leq & \max _{t \in I}|(F x)(t)-(F y)(t)| \\
\leq & L_{1} M_{2}\|x-y\|+L_{2} M_{1}\|x-y\| \\
\leq & \left(L_{1} M_{2}+L_{2} M_{1}\right)\|x-y\| \\
\leq & h\|x-y\| .
\end{aligned}
$$


Since

$$
h=\left(L_{1} M_{2}+L_{2} M_{1}\right)<1,
$$

then $F$ is a contraction and $F$ has a unique fixed point in $S$, thus there exists a unique solution for (1).

\section{Method of successive approximations (Picard method)}

Applying Picard method to the quadratic integral equation (1), the solution is constructed by the sequence

$$
\begin{aligned}
& x_{n}(t)=a(t)+g\left(t, x_{n-1}(t)\right) \int_{0}^{t} f\left(s, x_{n-1}(s)\right) d s, \quad n=1,2, \ldots, \\
& x_{0}(t)=a(t) .
\end{aligned}
$$

All the functions $x_{n}(t)$ are continuous functions and $x_{n}$ can be written as a sum of successive differences:

$$
x_{n}=x_{0}+\sum_{j=1}^{n}\left(x_{j}-x_{j-1}\right),
$$

This means that convergence of the sequence $x_{n}$ is equivalent to convergence of the infinite series $\sum\left(x_{j}-x_{j-1}\right)$ and the solution will be,

$$
x(t)=\lim _{n \rightarrow \infty} x_{n}(t),
$$

i.e. if the infinite series $\sum\left(x_{j}-x_{j-1}\right)$ converges, then the sequence $x_{n}(t)$ will converge to $x(t)$. To prove the uniform convergence of $\left\{x_{n}(t)\right\}$, we shall consider the associated series

$$
\sum_{n=1}^{\infty}\left[x_{n}(t)-x_{n-1}(t)\right] .
$$

From (2) for $n=1$, we get

$$
x_{1}(t)-x_{0}(t)=g\left(t, x_{0}(t)\right) \int_{0}^{t} f\left(s, x_{0}(s)\right) d s
$$

and

$$
\left|x_{1}(t)-x_{0}(t)\right| \leq M_{1} M_{2} \int_{0}^{t} d s=M_{1} M_{2} t .
$$


Now, we shall obtain an estimate for $x_{n}(t)-x_{n-1}(t), n \geq 2$

$$
\begin{aligned}
x_{n}(t) & -x_{n-1}(t) \leq g\left(t, x_{n-1}(t)\right) \int_{0}^{t} f\left(s, x_{n-1}(s)\right) d s \\
- & g\left(t, x_{n-2}(t)\right) \int_{0}^{t} f\left(s, x_{n-2}(s)\right) d s+g\left(t, x_{n-1}(t)\right) \\
\times & \int_{0}^{t} f\left(s, x_{n-2}(s)\right) d s-g\left(t, x_{n-1}(t)\right) \int_{0}^{t} f\left(s, x_{n-2}(s)\right) d s \\
\leq & g\left(t, x_{n-1}(t)\right) \int_{0}^{t}\left[f\left(s, x_{n-1}(s)\right)-f\left(s, x_{n-2}(s)\right)\right] d s \\
+ & {\left[g\left(t, x_{n-1}(t)\right)-g\left(t, x_{n-2}(t)\right)\right] \int_{0}^{t} f\left(s, x_{n-2}(s)\right) d s, }
\end{aligned}
$$

using assumptions (ii) and (iii), we get

$$
\begin{gathered}
\left|x_{n}(t)-x_{n-1}(t)\right| \leq M_{1} L_{2} \int_{0}^{t}\left|x_{n-1}(s)-x_{n-2}(s)\right| d s \\
+M_{2} L_{1}\left|x_{n-1}(t)-x_{n-2}(t)\right| \int_{0}^{t} d s .
\end{gathered}
$$

Putting $n=2$, then using (3) we get

$$
\begin{aligned}
\left|x_{2}(t)-x_{1}(t)\right| & \leq M_{1} L_{2} \int_{0}^{t}\left|x_{1}(s)-x_{0}(s)\right| d s+M_{2} L_{1}\left|x_{1}(t)-x_{0}(t)\right| t . \\
\left|x_{2}-x_{1}\right| & \leq M_{1}^{2} M_{2} L_{2} \frac{t^{2}}{2}+M_{1} M_{2}^{2} L_{1} t^{2} \\
& \leq M_{1} M_{2}\left(\frac{1}{2} M_{1} L_{2}+M_{2} L_{1}\right) t^{2} \\
\left|x_{3}-x_{2}\right| & \leq M_{1} L_{2} \int_{0}^{t}\left|x_{2}(s)-x_{1}(s)\right| d s+M_{2} L_{1}\left|x_{2}(t)-x_{1}(t)\right| \int_{0}^{t} d s \\
& \leq M_{1} M_{2}\left(\frac{1}{2} M_{1} L_{2}+M_{2} L_{1}\right)\left(\frac{1}{3} M_{1} L_{2}+M_{2} L_{1}\right) t^{3} .
\end{aligned}
$$

Repeating this technique, we obtain the general estimate for the terms of the series:

$$
\begin{aligned}
& \left|x_{n}-x_{n-1}\right| \\
& \quad \leq M_{1} M_{2}\left(\frac{1}{2} M_{1} L_{2}+M_{2} L_{1}\right)\left(\frac{1}{3} M_{1} L_{2}+M_{2} L_{1}\right) \times \cdots \times\left(\frac{1}{n} M_{1} L_{2}+M_{2} L_{1}\right) t^{n}
\end{aligned}
$$




$$
\begin{aligned}
& \leq M_{1} M_{2}\left(\frac{1}{2} M_{1} L_{2}+M_{2} L_{1}\right)\left(\frac{1}{3} M_{1} L_{2}+M_{2} L_{1}\right) \times \cdots \times\left(\frac{1}{n} M_{1} L_{2}+M_{2} L_{1}\right) \\
& \leq M_{1} M_{2}\left(M_{1} L_{2}+M_{2} L_{1}\right)\left(M_{1} L_{2}+M_{2} L_{1}\right) \times \cdots \times\left(M_{1} L_{2}+M_{2} L_{1}\right) \\
& \leq\left(M_{1} L_{2}+M_{2} L_{1}\right)^{n} .
\end{aligned}
$$

Since $\left(L_{1} M_{2}+L_{2} M_{1}\right)<1$, then the uniform convergence of

$$
\sum_{n=1}^{\infty}\left[x_{n}(t)-x_{n-1}(t)\right]
$$

is proved and so the sequence $\left\{x_{n}(t)\right\}$ is uniformly convergent.

Since $f(t, x)$ and $g(t, x)$ are continuous in $x$, then

$$
\begin{aligned}
x(t) & =\lim _{n \rightarrow \infty} g\left(t, x_{n}(t)\right) \int_{0}^{t} f\left(s, x_{n}(s)\right) d s \\
& =g(t, x(t)) \int_{0}^{t} f(s, x(s)) d s .
\end{aligned}
$$

thus, the existence of a solution is proved.

To prove the uniqueness, let $y(t)$ be a continuous solution of (1). Then

$$
y(t)=a(t)+g(t, y(t)) \int_{0}^{t} f(s, y(s)) d s \quad t \in[0,1]
$$

and

$$
\begin{aligned}
& y(t)-x_{n}(t) \leq g(t, y(t)) \int_{0}^{t} f(s, y(s)) d s-g\left(t, x_{n-1}(t)\right) \int_{0}^{t} f\left(s, x_{n-1}(s)\right) d s \\
& +g(t, y(t)) \int_{0}^{t} f\left(s, x_{n-1}(s)\right) d s-g(t, y(t)) \int_{0}^{t} f\left(s, x_{n-1}(s)\right) d s \\
& =g(t, y(t)) \int_{0}^{t}\left[f(s, y(s))-f\left(s, x_{n-1}(s)\right)\right] d s \\
& +\left[g(t, y(t))-g\left(t, x_{n-1}(t)\right)\right] \int_{0}^{t} f\left(s, x_{n-1}(s)\right) d s,
\end{aligned}
$$

using assumptions (ii) and (iii), we get

$$
\begin{gathered}
\left|y(t)-x_{n}(t)\right| \leq M_{1} L_{2} \\
\times \int_{0}^{t}\left|y(s)-x_{n-1}(s)\right| d s+M_{2} L_{1}\left|y(t)-x_{n-1}(t)\right| \int_{0}^{t} d s .
\end{gathered}
$$


But

$$
|y(t)-a(t)| \leq M_{1} M_{2} t
$$

and using (4) then we get

$$
\left|y(t)-x_{n}(t)\right| \leq\left(M_{1} L_{2}+M_{2} L_{1}\right)^{n} .
$$

Hence

$$
\lim _{n \rightarrow \infty} x_{n}(t)=y(t)=x(t)
$$

which completes the proof.

When $g(t, x)=1$, then $M_{1}=1$ and $L_{1}=0$ and we obtain the original Picard theorem [14] and [15].

Corollary 1. Let the assumptions of Theorem 1 (with $g(t, x)=1$ ) be satisfied. If $L_{2}<1$, then the integral equation

$$
x(t)=x_{0}(t)+\int_{0}^{t} f(s, x(s)) d s
$$

has a unique continuous solution.

\section{Adomian Decomposition Method (ADM)}

The Adomian decomposition method (ADM) is a nonnumerical method for solving a wide variety of functional equations and usually gets the solution in a series form.

Since the beginning of the 1980s, Adomian ([1-6] and [12-13]) has presented and developed a so-called decomposition method for solving algebraic, differential, integro-differential, differential-delay, and partial differential equations. The solution is found as an infinite series which converges rapidly to accurate solutions. The method has many advantages over the classical techniques, mainly, it makes unnecessary the linearization, perturbation and other restrictive methods and assumptions which may change the problem being solved, sometimes seriously. In recent decades, there has been a great deal of interest in the Adomian decomposition method. The method was successfully applied to 
a large amount of applications in applied sciences. For more details about the method and its application, see ([1-6] and [12-13]).

In this section, we shall study Adomian decomposition method (ADM) for the quadratic integral equation (1).

The solution algorithm of the quadratic integral equation (1) using ADM is,

$$
\begin{aligned}
& x_{0}(t)=a(t), \\
& x_{i}(t)=A_{(i-1)}(t) \int_{0}^{t} B_{(i-1)}(s) d s .
\end{aligned}
$$

where $A_{i}$ and $B_{i}$ are Adomian polynomials of the nonlinear terms $g(t, x)$ and $f(s, x)$ respectively, which have the form

$$
\begin{aligned}
& A_{n}=\frac{1}{n !} \frac{d^{n}}{d \lambda^{n}}\left[f\left(t, \sum_{i=0}^{\infty} \lambda^{i} x_{i}\right)\right]_{\lambda=0}, \\
& B_{n}=\frac{1}{n !} \frac{d^{n}}{d \lambda^{n}}\left[g\left(t, \sum_{i=0}^{\infty} \lambda^{i} x_{i}\right)\right]_{\lambda=0}
\end{aligned}
$$

and the solution will be,

$$
x(t)=\sum_{i=0}^{\infty} x_{i}(t)
$$

\subsection{Convergence analysis}

Theorem 2. Let the solution of the QIE (1) exists. If $\left|x_{1}(t)\right|<l, l$ is a positive constant, then the series solution (9) of the QIE (1) using ADM converges.

Proof. Define the sequence $\left\{S_{p}\right\}$ such that,

$$
S_{p}=\sum_{i=0}^{p} x_{i}(t)
$$

is the sequence of partial sums from the series solution $\sum_{i=0}^{\infty} x_{i}(t)$, and we have

$$
\begin{aligned}
& g(t, x)=\sum_{i=0}^{\infty} A_{i}, \\
& f(s, x)=\sum_{i=0}^{\infty} B_{i} .
\end{aligned}
$$


Let $S_{p}$ and $S_{q}$ be two arbitrary partial sums with $p>q$. Now, we are going to prove that $\left\{S_{p}\right\}$ is a Cauchy sequence in this Banach space $E$.

$$
\begin{aligned}
& S_{p}-S_{q}=\sum_{i=0}^{p} x_{i}-\sum_{i=0}^{q} x_{i} \\
& =\sum_{i=0}^{p} A_{(i-1)}(t) \int_{0}^{t} \sum_{i=0}^{p} B_{(i-1)}(s) d s-\sum_{i=0}^{q} A_{(i-1)}(t) \int_{0}^{t} \sum_{i=0}^{q} B_{(i-1)}(s) d s \\
& =\sum_{i=0}^{p} A_{(i-1)}(t) \int_{0}^{t} \sum_{i=0}^{p} B_{(i-1)}(s) d s-\sum_{i=0}^{q} A_{(i-1)}(t) \int_{0}^{t} \sum_{i=0}^{p} B_{(i-1)}(s) d s \\
& +\sum_{i=0}^{q} A_{(i-1)}(t) \int_{0}^{t} \sum_{i=0}^{p} B_{(i-1)}(s) d s-\sum_{i=0}^{q} A_{(i-1)}(t) \int_{0}^{t} \sum_{i=0}^{q} B_{(i-1)}(s) d s \\
& =\left[\sum_{i=0}^{p} A_{(i-1)}(t)-\sum_{i=0}^{q} A_{(i-1)}(t)\right] \int_{0}^{t} \sum_{i=0}^{p} B_{(i-1)}(s) d s \\
& +\sum_{i=0}^{q} A_{(i-1)}(t) \int_{0}^{t}\left[\sum_{i=0}^{p} B_{(i-1)}(s)-\sum_{i=0}^{q} B_{(i-1)}(s)\right] d s \\
& \left\|S_{p}-S_{q}\right\| \leq \max _{t \in I}\left|\sum_{i=q+1}^{p} A_{(i-1)}(t) \int_{0}^{t} \sum_{i=0}^{p} B_{(i-1)}(s) d s\right| \\
& +\max _{t \in I}\left|\sum_{i=0}^{q} A_{(i-1)}(t) \int_{0}^{t} \sum_{i=q+1}^{p} B_{(i-1)}(s) d s\right| \\
& \leq \max _{t \in I}\left|\sum_{i=q}^{p-1} A_{i}(t)\right| \int_{0}^{t}\left|\sum_{i=0}^{p} B_{(i-1)}(s) d s\right| d s \\
& +\max _{t \in I}\left|\sum_{i=0}^{q} A_{(i-1)}(t)\right| \int_{0}^{t}\left|\sum_{i=q}^{p-1} B_{i}(s)\right| d s \\
& \leq \max _{t \in I}\left|g\left(t, S_{p-1}\right)-g\left(t, S_{q-1}\right)\right| \int_{0}^{t}\left|f\left(t, S_{p}\right)\right| d s \\
& +\max _{t \in I}\left|g\left(t, S_{q}\right)\right| \int_{0}^{t}\left|f\left(t, S_{p-1}\right)-f\left(t, S_{q-1}\right)\right| d s \\
& \leq L_{1} M_{2} \max _{t \in I}\left|S_{p-1}-S_{q-1}\right|+L_{2} M_{1} \max _{t \in I}\left|S_{p-1}-S_{q-1}\right| \\
& \leq h \quad\left\|S_{p-1}-S_{q-1}\right\|
\end{aligned}
$$


Let $p=q+1$ then,

$$
\left\|S_{q+1}-S_{q}\right\| \leq h\left\|S_{q}-S_{q-1}\right\| \leq h^{2}\left\|S_{q-1}-S_{q-2}\right\| \leq \cdots \leq h^{q}\left\|S_{1}-S_{0}\right\|
$$

From the triangle inequality we have,

$$
\begin{aligned}
\left\|S_{p}-S_{q}\right\| & \leq\left\|S_{q+1}-S_{q}\right\|+\left\|S_{q+2}-S_{q+1}\right\|+\cdots+\left\|S_{p}-S_{p-1}\right\| \\
& \leq\left[h^{q}+h^{q+1}+\cdots+h^{p-1}\right]\left\|S_{1}-S_{0}\right\| \\
& \leq h^{q}\left[1+h+\cdots+h^{p-q-1}\right]\left\|S_{1}-S_{0}\right\| \\
& \leq h^{q}\left[\frac{1-h^{p-q}}{1-h}\right]\left\|x_{1}(t)\right\|
\end{aligned}
$$

Now $0<h<1$, and $p>q$ implies that $\left(1-h^{p-q}\right) \leq 1$. Consequently,

$$
\begin{aligned}
\left\|S_{p}-S_{q}\right\| & \leq \frac{h^{q}}{1-h}\left\|x_{1}(t)\right\| \\
& \leq \frac{h^{q}}{1-h} \max _{t \in I}\left|x_{1}(t)\right|
\end{aligned}
$$

but, $\left|x_{1}(t)\right|<l$ and as $q \rightarrow \infty$ then, $\left\|S_{p}-S_{q}\right\| \rightarrow 0$ and hence, $\left\{S_{p}\right\}$ is a Cauchy sequence in this Banach space $E$ and the series $\sum_{i=0}^{\infty} x_{i}(t)$ converges.

\subsection{Error analysis}

Theorem 3. The maximum absolute truncation error of the series solution (9) to the problem (1) is estimated to be,

$$
\max _{t \in I}\left|x(t)-\sum_{i=0}^{q} x_{i}(t)\right| \leq \frac{h^{q}}{1-h} \max _{t \in I}\left|x_{1}(t)\right|
$$

Proof. From Theorem 2 we have,

$$
\left\|S_{p}-S_{q}\right\| \leq \frac{h^{q}}{1-h} \max _{t \in I}\left|x_{1}(t)\right|
$$

but, $S_{p}=\sum_{i=0}^{p} x_{i}(t)$ as $p \rightarrow \infty$ then $S_{p} \rightarrow x(t)$ so,

$$
\left\|x(t)-S_{q}\right\| \leq \frac{h^{q}}{1-h} \max _{t \in I}\left|x_{1}(t)\right|
$$


so, the maximum absolute truncation error in the interval $I$ is,

$$
\max _{t \in I}\left|x(t)-\sum_{i=0}^{q} x_{i}(t)\right| \leq \frac{h^{q}}{1-h} \max _{t \in I}\left|x_{1}(t)\right|
$$

and this completes the proof.

\section{Numerical Examples}

In this section, we shall study some numerical examples and applying Picard and ADM methods, then comparing the results.

Example 1. Consider the following nonlinear QIE,

$$
x(t)=\left(t^{2}-\frac{t^{10}}{35}\right)+\frac{t}{5} x(t) \int_{0}^{t} s^{2} x^{2}(s) d s,
$$

and has the exact solution $x(t)=t^{2}$.

Applying Picard method to equation (10), we get

$$
\begin{aligned}
& x_{n}(t)=\left(t^{2}-\frac{t^{10}}{35}\right)+\frac{t}{5} x_{n-1}(t) \int_{0}^{t} s^{2} x_{n-1}^{2}(s) d s, \quad n=1,2, \ldots, \\
& x_{0}(t)=\left(t^{2}-\frac{t^{10}}{35}\right) .
\end{aligned}
$$

and the solution will be,

$$
x(t)=\lim _{n \rightarrow \infty} x_{n}(t) .
$$

Applying ADM to equation (10), we get

$$
\begin{aligned}
x_{0}(t) & =\left(t^{2}-\frac{t^{10}}{35}\right), \\
x_{i}(t) & =\frac{t}{5} x_{i-1}(t) \int_{0}^{t} s^{2} A_{i-1}(s) d s, \quad i \geq 1 .
\end{aligned}
$$

where $A_{i}$ are Adomian polynomials of the nonlinear term $x^{2}$, and the solution will be,

$$
x(t)=\sum_{i=0}^{q} x_{i}(t)
$$

Table 1 shows a comparison between the absolute error of Picard (when $n=5$ ) and ADM solutions (when $q=5$ ). 


\begin{tabular}{|c|c|c|}
\hline$t$ & $\left|x_{\text {Exact }}-x_{\text {Picard }}\right|$ & $\left|x_{\text {Exact }}-x_{\mathrm{ADM}}\right|$ \\
\hline 0.1 & 0 & $1.57823 \times 10^{-19}$ \\
\hline 0.2 & 0 & $1.03431 \times 10^{-14}$ \\
\hline 0.3 & $2.94109 \times 10^{-22}$ & $6.79375 \times 10^{-12}$ \\
\hline 0.4 & 0 & $6.77831 \times 10^{-10}$ \\
\hline 0.5 & 0 & $2.40789 \times 10^{-8}$ \\
\hline 0.6 & $1.50584 \times 10^{-19}$ & $4.44997 \times 10^{-7}$ \\
\hline 0.7 & $1.57984 \times 10^{-16}$ & $5.23525 \times 10^{-6}$ \\
\hline 0.8 & $9.63707 \times 10^{-14}$ & 0.0000441862 \\
\hline 0.9 & $2.73899 \times 10^{-11}$ & 0.000288498 \\
\hline 1 & $4.26772 \times 10^{-9}$ & 0.00153008 \\
\hline
\end{tabular}

Table 1 - Absolute error.

Example 2. Consider the following nonlinear QIE,

$$
x(t)=\left(t^{3}-\frac{t^{19}}{100}-\frac{t^{20}}{110}\right)+\frac{t^{3}}{10} x^{2}(t) \int_{0}^{t}(s+1) x^{3}(s) d s,
$$

and has the exact solution $x(t)=t^{3}$.

Applying Picard method to equation (11), we get

$$
\begin{aligned}
& x_{n}(t)=\left(t^{3}-\frac{t^{19}}{100}-\frac{t^{20}}{110}\right)+\frac{t^{3}}{10} x_{n-1}^{2}(t) \int_{0}^{t}(s+1) x_{n-1}^{3}(s) d s, \quad n=1,2, \ldots, \\
& x_{0}(t)=\left(t^{3}-\frac{t^{19}}{100}-\frac{t^{20}}{110}\right) .
\end{aligned}
$$

and the solution will be,

$$
x(t)=\lim _{n \rightarrow \infty} x_{n}(t) .
$$

Applying ADM to equation (11), we get

$$
\begin{aligned}
& x_{0}(t)=\left(t^{3}-\frac{t^{19}}{100}-\frac{t^{20}}{110}\right) \\
& x_{i}(t)=\frac{t^{3}}{10} A_{i-1}(t) \int_{0}^{t}(s+1) B_{i-1}(s) d s, \quad i \geq 1 .
\end{aligned}
$$


where $A_{i}$ and $B_{i}$ are Adomian polynomials of the nonlinear terms $x^{2}$ and $x^{3}$ respectively, and the solution will be,

$$
x(t)=\sum_{i=0}^{q} x_{i}(t)
$$

Table 2 shows a comparison between the absolute error of Picard (when $n=3$ ) and ADM solutions (when $q=3$ ).

\begin{tabular}{|c|c|c|}
\hline$t$ & $\left|x_{\text {Exact }}-x_{\text {Picard }}\right|$ & $\left|x_{\text {Exact }}-x_{\mathrm{ADM}}\right|$ \\
\hline 0.1 & $1.88079 \times 10^{-34}$ & $1.88079 \times 10^{-34}$ \\
\hline 0.2 & $2.46519 \times 10^{-29}$ & $1.89203 \times 10^{-26}$ \\
\hline 0.3 & $2.24387 \times 10^{-26}$ & $9.48288 \times 10^{-21}$ \\
\hline 0.4 & $1.61559 \times 10^{-24}$ & $1.08415 \times 10^{-16}$ \\
\hline 0.5 & 0 & $1.55749 \times 10^{-13}$ \\
\hline 0.6 & $5.39199 \times 10^{-21}$ & $6.01199 \times 10^{-11}$ \\
\hline 0.7 & $1.90012 \times 10^{-16}$ & $9.35496 \times 10^{-9}$ \\
\hline 0.8 & $1.21415 \times 10^{-12}$ & $7.47272 \times 10^{-7}$ \\
\hline 0.9 & $2.79233 \times 10^{-9}$ & 0.0000356866 \\
\hline 1 & $2.82633 \times 10^{-6}$ & 0.0011119 \\
\hline
\end{tabular}

Table 2 - Absolute error.

Example 3. Consider the following nonlinear QIE [9],

$$
x(t)=t^{3}+\left(\frac{1}{4} x(t)+\frac{1}{4}\right)\left(\int_{0}^{t} t+\cos \left(\frac{x(s)}{1+x^{2}(s)}\right)\right) d s,
$$

Applying Picard method to equation (12), we get

$x_{n}(t)=t^{3}+\left(\frac{1}{4} x_{n-1}(t)+\frac{1}{4}\right)\left(\int_{0}^{t} t+\cos \left(\frac{x_{n-1}(s)}{1+x_{n-1}^{2}(s)}\right)\right) d s, \quad n=1,2, \ldots$,

$x_{0}(t)=t^{3}$.

and the solution will be,

$$
x(t)=x_{n}(t) .
$$


Applying ADM to equation (12), we get

$$
\begin{aligned}
x_{0}(t) & =t^{3}, \\
x_{n+1}(t) & =\left(\frac{1}{4} x_{n}(t)+\frac{1}{4}\right)\left(\int_{0}^{t} t+A_{i-1}(s)\right) d s, \quad i \geq 1 .
\end{aligned}
$$

where $A_{i}$ are Adomian polynomials of the nonlinear term $\cos \left(\frac{x(s)}{1+x^{2}(s)}\right)$ and the solution will be,

$$
x(t)=\sum_{i=0}^{q} x_{i}(t) .
$$

Table 3 shows a caparison between ADM and Picard solutions.

\begin{tabular}{|c|c|c|c|}
\hline$t$ & ADM solution & Picard solution & $\left|x_{\text {ADM }}-x_{\text {Picard }}\right|$ \\
\hline 0.1 & 0.0285275 & 0.0272762 & 0.00125125 \\
\hline 0.2 & 0.0684798 & 0.0634398 & 0.00504 \\
\hline 0.3 & 0.127128 & 0.115575 & 0.0115538 \\
\hline 0.4 & 0.212929 & 0.191649 & 0.02128 \\
\hline 0.5 & 0.335783 & 0.300627 & 0.0351563 \\
\hline 0.6 & 0.507262 & 0.452542 & 0.05472 \\
\hline 0.7 & 0.740798 & 0.65854 & 0.0822587 \\
\hline 0.8 & 1.05195 & 0.930992 & 0.12096 \\
\hline 0.9 & 1.45895 & 1.28389 & 0.175061 \\
\hline 1 & 1.98343 & 1.73343 & 0.25 \\
\hline
\end{tabular}

Table 3 - Absolute error.

Example 4. Consider the following nonlinear QIE [8],

$$
x(t)=e^{-t}+x(t) \int_{0}^{t} \frac{t^{2} \ln (1+s|x(s)|)}{2 e^{(t+s)}} d s, \quad 0<t \leq 2 .
$$

Applying Picard method to equation (13), we get

$$
\begin{aligned}
x_{n}(t) & =e^{-t}+x_{n-1}(t) \int_{0}^{t} \frac{t^{2} \ln \left(1+s\left|x_{n-1}(s)\right|\right)}{2 e^{(t+s)}} d s, \quad n=1,2, \ldots, x_{0}(t) \\
& =e^{-t} .
\end{aligned}
$$

and the solution will be,

$$
x(t)=x_{n}(t)
$$


Applying ADM to equation (13), we get

$$
\begin{aligned}
x_{0}(t) & =e^{-t}, \\
x_{n+1}(t) & =x_{n}(t) \int_{0}^{t} \frac{t^{2} A_{i-1}(s)}{2 e^{(t+s)}} d s, \quad i \geq 1 .
\end{aligned}
$$

where $A_{i}$ are Adomian polynomials of the nonlinear term $\ln (1+s|x(s)|)$ and the solution will be,

$$
x(t)=\sum_{i=0}^{q} x_{i}(t) .
$$

Table 4 shows a caparison between ADM and Picard solutions.

\begin{tabular}{|c|c|c|c|}
\hline$t$ & ADM solution & Picard solution & $\left|x_{\text {ADM }}-x_{\text {Picard }}\right|$ \\
\hline 0.2 & 0.818926 & 0.818926 & $1.11022 \times 10^{-16}$ \\
\hline 0.4 & 0.671897 & 0.671897 & 0 \\
\hline 0.6 & 0.552921 & 0.552921 & 0 \\
\hline 0.8 & 0.456136 & 0.456136 & 0 \\
\hline 1 & 0.376724 & 0.376724 & 0 \\
\hline 1.2 & 0.31109 & 0.31109 & 0 \\
\hline 1.4 & 0.256612 & 0.256612 & $2.77556 \times 10^{-17}$ \\
\hline 1.6 & 0.211338 & 0.211338 & 0 \\
\hline 1.8 & 0.173748 & 0.173748 & 0 \\
\hline 2 & 0.142602 & 0.142602 & 0 \\
\hline
\end{tabular}

Table 4 - Absolute error.

\section{Conclusion}

We used two analytical methods to solve QIEs; Picard method and ADM, from the results in the tables we see that Picard method gives more accurate solution than ADM.

\section{REFERENCES}

[1] G. Adomian, Stochastic System. Academic Press (1983).

[2] G. Adomian, Nonlinear Stochastic Operator Equations. Academic Press, San Diego (1986). 
[3] G. Adomian, Nonlinear Stochastic Systems: Theory and Applications to Physics. Kluwer (1989).

[4] G. Adomian, R. Rach and R. Mayer, Modified decomposition. J. Appl. Math. Comput., 23 (1992), 17-23.

[5] K. Abbaoui and Y. Cherruault, Convergence of Adomian's method Applied to Differential Equations. Computers Math. Applic., 28 (1994) 103-109.

[6] G. Adomian, Solving Frontier Problems of Physics: The Decomposition Method. Kluwer (1995).

[7] J. Banaś, M. Lecko and W.G. El-Sayed, Existence Theorems of Some Quadratic Integral Equation. J. Math. Anal. Appl., 227 (1998), 276-279.

[8] J. Banaś and A. Martinon, Monotonic Solutions of a quadratic Integral Equation of Volterra Type. Comput. Math. Appl., 47 (2004), 271-279.

[9] J. Banaś, J. Caballero, J. Rocha and K. Sadarangani, Monotonic Solutions of a Class of Quadratic Integral Equations of Volterra Type. Computers and Mathematics with Applications, 49 (2005), 943-952.

[10] J. Banaś, J. Rocha Martin and K. Sadarangani, On the solution of a quadratic integral equation of Hammerstein type. Mathematical and Computer Modelling, 43 (2006), 97-104.

[11] J. Banaś and B. Rzepka, Monotonic solutions of a quadratic integral equations of fractional order. J. Math. Anal. Appl., 332 (2007), 1370-11378.

[12] Y. Cherruault, Convergence of Adomian method. Kybernetes, 18 (1989), 31-38.

[13] Y. Cherruault, G. Adomian, K. Abbaoui and R. Rach, Further remarks on convergence of decomposition method. Int. J. of Bio-Medical Computing., 38 (1995), 89-93.

[14] R.F. Curtain and A.J. Pritchard, Functional Analysis in Modern Applied Mathematics. Academic Press (1977).

[15] C. Corduneanu, Principles of Differential and integral equations. Allyn and Bacon. Hnc., New York (1971).

[16] A.M.A. El-Sayed, M.M. Saleh and E.A.A. Ziada, Numerical and Analytic Solution for Nonlinear Quadratic Integral Equations. Math. Sci. Res. J., 12(8) (2008), 183-191.

[17] A.M.A. El-Sayed and H.H.G. Hashem, Carathéodory type theorem for a nonlinear quadratic integral equation. Math. Sci. Res. J., 12(4) (2008), 71-95.

[18] A.M.A. El-Sayed and H.H.G. Hashem, Integrable and continuous solutions of nonlinear quadratic integral equation. Electronic Journal of Qualitative Theory of Differential Equations, 25 (2008), 1-10.

[19] A.M.A. El-Sayed and H.H.G. Hashem, Monotonic positive solution of nonlinear quadratic Hammerstein and Urysohn functional integral equations. Commentationes Mathematicae, 48(2) (2008), 199-207. 
[20] A.M.A. El-Sayed and H.H.G. Hashem, Weak maximal and minimal solutions for Hammerstein and Urysohn integral equations in reflexive Banach spaces. Differential Equation and Control Processes, 4 (2008), 50-62.

[21] A.M.A. El-Sayed and H.H.G. Hashem, Monotonic solutions of functional integral and differential equations of fractional order. E.J. Qualitative Theory of Diff. Equ., 7 (2009), $1-8$.

[22] A.M.A. El-Sayed and H.H.G. Hashem, Solvability of nonlinear Hammerstein quadratic integral equations. J. Nonlinear Sci. Appl., 2(3) (2009), 152-160. 